\title{
Late Cutaneous Schistosomiasis Representing an Isolated Skin Manifestation of Schistosoma mansoni Infection
}

\author{
G. Kick M. Schaller H.C. Korting \\ Department of Dermatology, Ludwig Maximilian University, Munich, Germany
}

\section{Key Words}

Cutaneous schistosomiasis $\cdot$ Schistosoma

mansoni $\cdot$ Bilharziasis cutanea tarda

\begin{abstract}
Ectopic late cutaneous schistosomiasis is usually preceded or accompanied by visceral schistosomiasis infection. Our patient presented the very rare case of late cutaneous schistosomiasis as an isolated skin manifestation. Perigenital lesions occurred 1 year after contact with infested water. Identification of the few eggs remaining in the late lesion among the dense cellular infiltrate was difficult. Electron-microscopic studies clearly demonstrated the characteristic eggshell ultrastructure.
\end{abstract}

Copyright (C) 2000 S. Karger AG, Basel

\section{Introduction}

The denomination schistosomiasis indicates a group of diseases caused by trematodes of the genus Schistosoma [1]. In particular, the life cycle of the species Schistosoma mansoni is in short as follows: after sexual reproduction of adult worms within the tributaries of the inferior mesenteric vein of humans, representing the definite hosts, females produce eggs, characterized by a typical lateral spine. Some of the eggs pass through the vessel wall into the intestine, then reaching the external world with the faeces. Contact with water induces the release of miracidia, which infect freshwater snails. Within these intermediate hosts, thousands of fork-tailed cercariae develop. Losing their tails, they quickly penetrate human skin, pass through the tissue as schistosomula and develop to male or female schistosomes. Clinical schistosomiasis mainly occurs in heavily infected individuals and is caused by egg deposition in the bowel, liver and lung, leading to a chronic granulomatous inflammation and fibrosis.

Intermittent abdominal pain, diarrhoea, hepatosplenomegaly and portal and pulmonary hypertension are consecutive symptoms. Other sites of oviposition, as presented by cutaneous lesions, are rare. Our case report demonstrates the clinical, histological and ultrastructural findings, linked to late cutaneous schistosomiasis.

\section{Case Report}

During a 1-year travel through Africa, a 35 -year-old man enjoyed swimming in Lake Malawi and the Niger River. One year later, he developed a slowly growing and initially slightly itching tumour in his right groin. There was no skin eruption and no history of haematuria or cystitis, bloody diarrhoea or dysentery during his stay in Africa. Physical examination showed a broadly based reddish tumour in the right groin, partly with a glassy surface (fig. 1). Regarding the clinical findings, pyogenous granuloma, squamous cell carcinoma, condylomata lata, condylomata acuminata or amoebiasis cutis had to be considered in the differential diagnosis.

The lesion was excised for histological evaluation. Pseudo-epitheliomatous hyper- plasia of the epidermis was found as a prominent feature. The dermis presented a dense infiltrate of eosinophils, lymphocytes and plasma cells. Surrounded by the cellular infiltrate, an egg of $S$. mansoni with a typical lateral spine and parts of a miracidium could be identified (fig. 2). Further investigation was performed by electron-microscopic studies. A dense granulomatous infiltrate was seen in the dermis comprising lymphocytes, histiocytes, multinucleate giant cells and large numbers of eosinophils. In the papillary dermis several $S$. mansoni eggs in different stages of development were present measuring $10-100 \mu \mathrm{m}$ in diameter. Immature, intermediately mature and mature eggs (fig. 3) were discriminated by the different composition of their egg shells and the interior of the eggs. Figure 4 demonstrates in de-

Fig. 1. Paragenital Schistosoma granuloma in the right groin.

Fig. 2. S. mansoni egg surrounded by an infiltrate of eosinophils, lymphocytes and plasma cells.

Fig. 3. S. mansoni egg in the dermis. Magnification $\times 12,500$.

Fig. 4. Composition of egg shell and envelope of mature S. mansoni egg in detail. Parts of the shell are the peripheral microspines of the outer layer (S), the middle layer with intermediate electron density and the inner layer with extreme electron density. The envelope consists of Reynolds' layer (R), the von Lichtenberg envelope (Li) and a fluid-filled cavity, Lehmann's lacuna (Le). Magnification $\times 29,000$. Bar $=1.0 \mu \mathrm{m}$.

\section{KARGER \\ Fax +4161306 1234 \\ E-Mail karger@karger.ch} www. karger.com (c) 2000 S. Karger AG, Basel

1018-8665/00/2002-0144\$17.50/0

Accessible online at: www. karger.com/journals/drm
Prof. Hans Christian Korting

Dermatologische Klinik und Poliklinik der Ludwig-Maximilians-Universität München Frauenlobstrasse 9-11

D-80337 Munich (Germany)

Tel. +49895160 4615, Fax +498951604602 

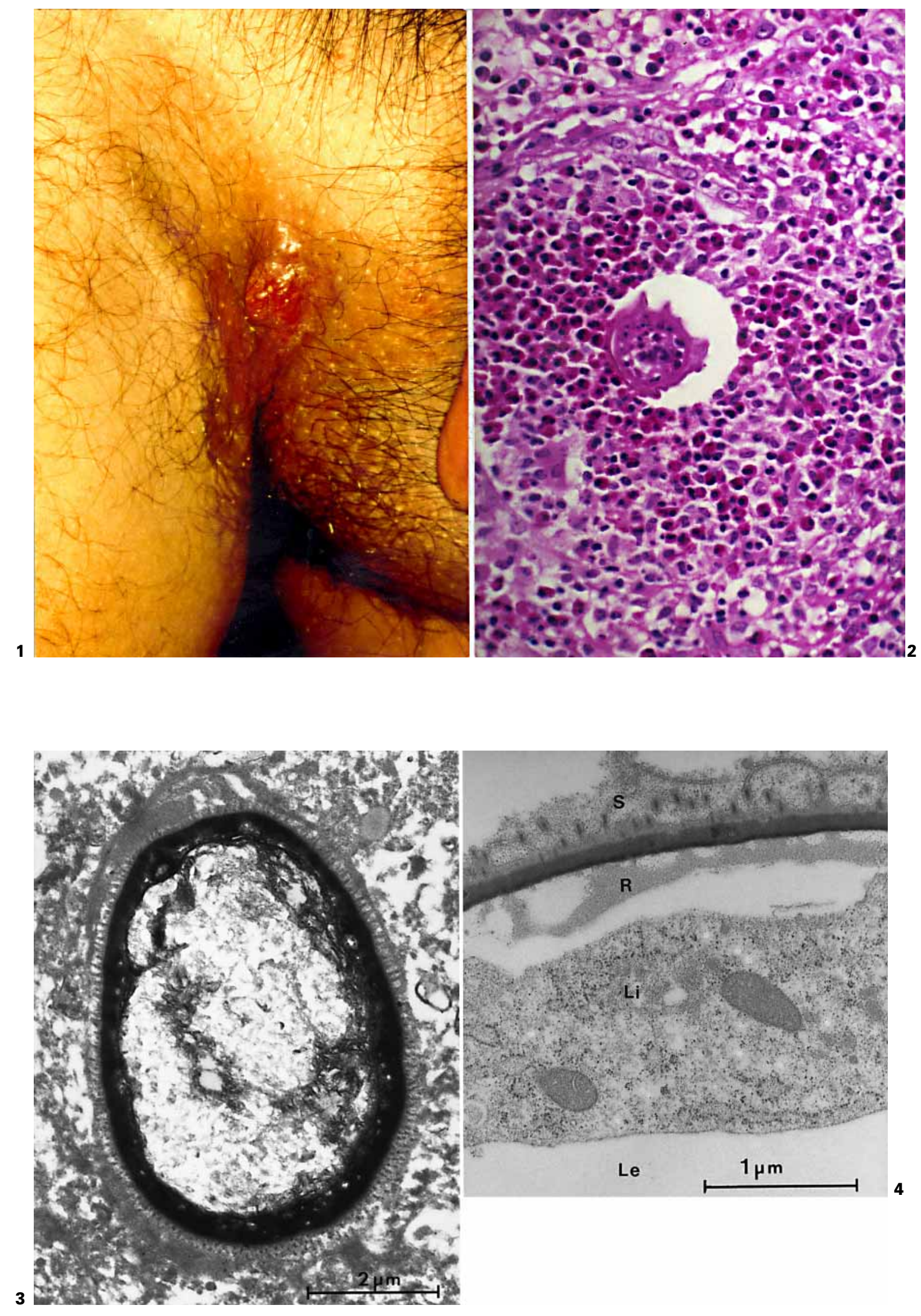

Late Cutaneous Schistosomiasis

Dermatology 2000;200:144-146 
tail the typical composition of the egg shell and the envelope of a mature $S$. mansoni egg near the pole. The shell is composed of three layers: the peripheral microspine layer, a middle layer with intermediate electron density and an inner layer with extreme electron density. The space between the developing miracidium and this inner electron-dense layer of the egg shell consists of three parts: Raynolds' layer, which lies immediately subjacent to the egg shell, the von Lichtenberg envelope, which consists of a single layer of flattened cells, and a fluid-filled cavity, Lehmann's lacuna, which surrounds the developing miracidium (not shown). The enzyme-linked immunosorbent assay (ELISA) against soluble egg antigen was positive, repeated stool and centrifuged urine examinations for eggs were negative. Abdominal sonography was normal. The patient was diagnosed as having bilharziasis cutanea tarda. Specific treatment was performed by peroral praziquantel $(2 \times 1.8 \mathrm{~g})$ for 1 day.

\section{Discussion}

Late cutaneous bilharziasis is a very rare manifestation of the most important human helminthic disease worldwide, which is schistosomiasis.

Originally this mammalian blood fluke infection was an African disease, later exported by the slave trade to Brazil, Venezuela and the Caribbean Islands [1]. Far from endemic areas, travelling habits in modern times constitute a diagnostic challenge for dermatologists and dermatohistopathologists in particular. The deposition of $S$. mansoni eggs in the dermis is mainly associated

\section{References}

1 World Health Organization: The control of schistosomiasis. WHO Tech Rep Ser 1985;728: 1-49.

2 Chaves E. Figueiredo JN: Schistosomiasis of the scrotum. Rev Inst Med Trop São Paulo 1965;7:343-345.

3 Farrell AM, Woodrow D, Bryceson AD, Bunker CB, Cream JJ: Ectopic cutaneous schistosomiasis: Extragenital involvement with progressive upward spread. Br J Dermatol 1996;135: 110-112.

4 Faust EC: An enquiry into the ectopic lesions in schistosomiasis. Am J Trop Med Hyg 1948; 28:175-199. with severe systemic invasion. Two types of lesions are distinguished, according to their location. The most common sites are the genital and perigenital areas (anus, perineum and groins). Schistosoma eggs reach this region by portosystemic anastomoses, presumably via the communication between the inferior mesenteric vein and the rectal venous plexus [2]. The second type, summarized as extragenital lesions mainly occurring on the trunk, is far less common than genital and paragenital lesions and the way of oviposition is not known [3-7]. Polyploid or vegetant tumours, papules or ulcerations are most common manifestations of both types of late cutaneous schistosomiasis. In the very rare cases of isolated skin manifestation without visceral disease, as presented by our patient, lesions are not easily diagnosed by clinical examination. Identification of schistosomal eggs is mandatory for the diagnosis of cutaneous bilharziasis. Although they are easily detected by the histopathologist at an early stage, eggs in late stages are rare and analysis of multiple layers of tissue is necessary to find eggs among the dense connective tissue. Granuloma formation is caused by a delayed-type T-cell-mediated hypersensitivity reaction against soluble egg antigens produced by miracidia and penetrating the eggshell [8]. The ectopic form of this parasitic disease seems to be relatively rare in tropic and subtropic regions with high endemicity probably due to natural immunity depending on genetic factors [7,9]. Accordingly, ectopic forms of schistosomiasis have been reported more frequently in Europeans visiting the endemic areas for a short time [1012]. Besides bilharziasis cutanea tarda, cercarial dermatitis and 'Katayama fever' are more common cutaneous manifestations of schistosomiasis. An itchy maculopapular rash after repeated exposure to cercariae is wellknown hypersensitivity skin reaction also named 'swimmer's itch' [13]. However, this reaction is mainly induced by avian cercariae penetrating human skin without further progression of their life cycle. The dermatitis is more intense than in cases of human schistosomal cercarial penetration and can occur in less than $15 \mathrm{~min}$ in sensitized humans.

Urticarial eruption, combined with acute febrile illness, eosinophilia, headache, diarrhoea and cough are typical signs of the 'acute toxaemic' schistosomiasis or 'Katayama syndrome'. This second cutaneous manifestation of schistosomiasis is thought to be immune complex mediated and appears suddenly 2-3 weeks after infection.

We here report the very rare case of a patient presenting isolated skin manifestations without any preceding manifestation involving the genitourinary or intestinal systems. Clinicians should be aware of this differential diagnosis even if the patient has returned from endemic areas months before the clinical manifestation. Finally identification of eggs in the dense connective tissue may present problems to dermatopathologists caused by the eggs' scarcity in the very late lesions.

\section{Acknowledgement}

We wish to thank Prof. P. Racz from the Bernhard Nocht Institute for Tropical Medicine, Department of Pathology, Hamburg, Germany, for the identification of S. mansoni eggs.
5 Jacyk WK, Lawande RV, Tulpule SS: Unusual presentation of extragenital cutaneous Schistosomiasis mansoni. Br J Dermatol 1980;103: 205-208.

6 Milligan A, Burns DA: Ectopic cutaneous schistosomiasis and schistosomal ocular inflammatory disease. Br J Dermatol 1988;119: 793-798.

7 Obasi OE: Cutaneous schistosomiasis in Nigeria: An update. Br J Dermatol 1986;114:597602.

8 Davis BH, Mahmoud AA, Warren KS: Granulomatous hypersensitivity to Schistosoma mansoni eggs in thymectomized and bursectomized chickens. J Immunol 1974; 113:1064-1067.
9 Abdel-Aziz AH: Cutaneous bilharzial granulomas. A histopathologic study. Cutis 1976;18: 516-519.

10 Findlay GH, Whiting DA: Disseminated and zosteriform cutaneous schistosomiasis. $\mathrm{Br} \mathrm{J}$ Dermatol 1971;85(suppl 7):98.

11 Saxe N, Gordon W: Schistosomiasis of spinal cord and skin. S Afr Med J 1975;49:57-58.

12 Wood MG, Srolovitz H, Schetman D: Schistosomiasis: Paraplegia and ectopic skin lesions as admission symptoms. Arch Dermatol 1976; 112:690-695.

13 Bastert J, Sing A, Wollenberg A, Korting HC: Aquarium dermatitis: Cercarial dermatitis in an aquarist. Dermatology 1998;197:84-86. 